\title{
A NOTE ON THE UNSTABILITY CONDITIONS OF THE STEENROD SQUARES ON THE POLYNOMIAL ALGEBRA
}

\author{
Ali Sarbaz Janfada
}

\begin{abstract}
We extend some results involved the action of the Steenrod operations on monomials and get some corollaries on the hit problem. Then, by multiplying some special matrices, we obtain an efficient tool to compute the action of these operations.
\end{abstract}

\section{Preliminaries}

In 1947, Steenrod [21] introduced the Steenrod squares $S q^{k}$ in terms of cocycles in simplicial cochain complex by modifying the Alexander-Ĉech-Whitney formula for the cup product construction. Serre [16] showed that they generate all stable operations in cohomology over $\mathbb{F}_{2}$ under composition. For an overview on algebraic topology we cite [4]. Cartan [2] discovered a formula for working out a Steenrod square on a product of cohomology classes $f, g$.

Theorem 1.1 (Cartan formula). $S q^{k}(f g)=\sum_{0 \leq r \leq k} S q^{r}(f) S q^{k-r}(g)$.

Adem [1] and Serre [16] established a faithful representation of $\mathcal{A}$ by its action on the cohomology of a test space consisting of infinite real projective spaces whose cohomology is the polynomial algebra $\mathbf{P}(n)=\mathbb{F}_{2}\left[x_{1}, x_{2}, \ldots, x_{n}\right]=$ $\bigoplus_{d \geq 0} \mathbf{P}^{d}(n)$, viewed as a graded module over the Steenrod algebra $\mathcal{A}$ at prime 2 . The grading is by the homogeneous polynomials $\mathbf{P}^{d}(n)$ of degree $d$ in the variables $x_{1}, x_{2}, \ldots, x_{n}$ of grading 1 . We cite to [5] and [11] in cohomology operations and to [11] and [22] in the Steenrod algebra.

The Steenrod algebra $\mathcal{A}$ is defined to be the graded algebra over the field $\mathbb{F}_{2}$, generated by the Steenrod squares $S q^{k}$, in grading $k \geq 0$, subject to the Adem relations [7, 24]. From a topological point of view, the Steenrod algebra is the algebra of stable cohomology operations for ordinary cohomology $\mathbf{H}^{*}$ over $\mathbb{F}_{2}$.

For present purpose we only need to know that the Steenrod algebra acts by composition of linear operators on $\mathbf{P}(n)$ and the action of the Steenrod squares

Received October 26, 2007; Revised May 24, 2008.

2000 Mathematics Subject Classification. 55S10.

Key words and phrases. Steenrod squares, hit problem.

This work was partially financed by grant No. 010/4/87.

(C)2009 The Korean Mathematical Society 
$S q^{k}: \mathbf{P}^{d}(n) \rightarrow \mathbf{P}^{d+k}(n)$ is determined by the Cartan formula and the following rules $[24]$.

Proposition 1.2. For homogeneous elements $f, g$ in $\mathbf{P}(n)$ we have

(i) $S q^{0}$ is the identity homomorphism;

(ii) $S q^{k}(f)=f^{2}$ if $\operatorname{deg}(f)=k$ and $S q^{k}(f)=0$ if $\operatorname{deg}(f)<k$.

(The statement (ii) is the so-called unstability conditions.)

The Cartan formula can be expressed in more concise form by defining total Steenrod square by $S q=S q^{0}+S q^{1}+\cdots$. This acts on $\mathbf{P}(n)$ since by the property (ii) in the above proposition, only a finite number of $S q^{k}$ 's can be nonzero on a given polynomial. The Cartan formula then says that $S q(f g)=$ $S q(f) S q(g)$, so $S q$ is a ring homomorphism $S q: \mathbf{P}(n) \rightarrow \mathbf{P}(n)$. Now, we can use $S q$ to compute the operator $S q^{k}$ via the following lemmas [22].

Lemma 1.3. If $\operatorname{deg}(x)=1$, then $S q^{k}\left(x^{\alpha}\right)=\left(\begin{array}{l}\alpha \\ k\end{array}\right) x^{k+\alpha}$ for any non negative integer $\alpha$.

The following lemma is now immediate.

Lemma 1.4. If $\operatorname{deg}(x)=1$, then

$$
S q^{k}\left(x^{2^{\tau}}\right)= \begin{cases}x^{2^{\tau}} & \text { if } k=0 ; \\ 0 & \text { if } 0<k<2^{\tau} ; \\ x^{2^{\tau+1}} & \text { if } k=2^{\tau} .\end{cases}
$$

Remark. It is clear by Proposition 1.2 that $S q^{k}\left(x^{2^{\tau}}\right)=0$ if $k>2^{\tau}$.

\section{Motivation, making the start point smooth}

On studies about Steenrod squares over polynomial algebras, the essence of work hangs on unstability conditions and Cartan formula. In such studies, we often handle with lower variables and lower degrees, as start point, and try to widen the gate more and more to get some results. The author has experienced this in $[7,8,10]$.

Our aim of this paper is to make this start point smooth. To this end, we first extend Lemma 1.4 (Lemmas 3.1 and 3.2) and deduce some useful tools in handling with the Steenrod operations. In particular, in Corollary 3.3, we show that given $\tau \geq 1$, the $S q^{k}\left(x_{1}^{\alpha_{1}} x_{2}^{\alpha_{2}} \cdots x_{n}^{\alpha_{n}}\right)$, where $1 \leq k<2^{\tau}$ and $1 \leq \alpha_{i} \leq 2^{\tau}$, determine all $S q^{\ell}\left(x_{1}^{\beta_{1}} x_{2}^{\beta_{2}} \cdots x_{n}^{\beta_{n}}\right)$ for any $\beta_{i} \geq 1$ and any $\ell$. On the other hand, if we change the places of $\alpha_{i}$ and $\alpha_{j}$ in $S q^{k}\left(x_{1}^{\alpha_{1}} x_{2}^{\alpha_{2}} \cdots x_{n}^{\alpha_{n}}\right)$, the results will be a permutation of $x_{i}$ and $x_{j}$. So, to handle with the $S q$ 's it is sufficient to know only the

$$
S q^{k}\left(x_{1}^{\alpha_{1}} x_{2}^{\alpha_{2}} \cdots x_{n}^{\alpha_{n}}\right) \text { with } \alpha_{1} \leq \alpha_{2} \leq \cdots \leq \alpha_{n} \leq 2^{\tau} \text { and } 1 \leq k<2^{\tau}
$$

for some $\tau>0$.

Then, as a corollary (Proposition 4.1), we obtain some hit monomials from the others. 
Finally, we calendar the road using a roller called $S q$-matrix. Let $1 \leq k \leq$ $2^{m}-1$, where $m$ is some positive integer. By the $S q$-matrix $\mathcal{S}_{k}$ we understand a $2^{m} \times(k+1)$ matrix defined by

$$
\left(\mathcal{S}_{k}\right)_{i j}=S q^{j-1}\left(x^{i}\right) .
$$

In Proposition 6.6 we shall show

$$
\left(\mathcal{S}_{k} \mathcal{S}_{k}^{r t}\right)_{i j}=S q^{k}\left(x^{i} y^{j}\right), \quad 1 \leq i, j \leq 2^{m}
$$

where $\mathcal{S}_{k}^{r t}$ is the transpose of $\mathcal{S}_{k}$ in reversed order. How do we use the $S q$ matrices to compute the Steenrod squares in two variables? Well, in lower degrees, we may use the matrix product in the relation (2). For example, assume that $\mathcal{S}_{6}$ is the matrix obtained by taking the first eight rows and the first seven columns of the matrix of Figure 2 (In Section 6 we shall exhibit a clear algorithm to form the $S q$-matrices). Then the relation (2) implies $\left(\mathcal{S}_{6} \mathcal{S}_{6}^{r t}\right)_{57}=$ $5 \cdot 13+6 \cdot 12+9 \cdot 9+10 \cdot 8$. This means $S q^{6}\left(x^{5} y^{7}\right)=x^{5} y^{13}+x^{6} y^{12}+x^{9} y^{9}+x^{10} y^{8}$.

In high degrees, one may save an $S q$-matrix of as big size as possible in some memories. Now, appealing to the definition (1) and the relation (2), one may write a simple program to compute the Steenrod squares $S q^{k}\left(x^{i} y^{j}\right)$, without any use to concerned softwares. For more than two variables, the above process can be extended inductively.

It is clear that working on monomials are so easier. On the other hand, polynomials are nothing but sums of monomials. That is why we do concentrate on monomials.

\section{Main results}

We shall adopt the following notations for any positive integer $\tau$.

$$
\begin{aligned}
\boldsymbol{x}^{\boldsymbol{\alpha}} & =x_{1}^{\alpha_{1}} x_{2}^{\alpha_{2}} \cdots x_{n}^{\alpha_{n}}, \\
\boldsymbol{x}^{\boldsymbol{m}\left(2^{\tau}\right)} & =x_{1}^{m_{1}\left(2^{\tau}\right)} x_{2}^{m_{2}\left(2^{\tau}\right)} \cdots x_{n}^{m_{n}\left(2^{\tau}\right)},
\end{aligned}
$$

where, for $1 \leq i \leq n$, the $\alpha_{i}$ and the $m_{i}$ are non negative integers.

The following lemma is an extension of Lemma 1.4.

\section{Lemma 3.1.}

$$
S q^{k}\left(\boldsymbol{x}^{2^{\tau}}\right)=\left\{\begin{array}{lll}
\text { (i) } & x^{2^{\tau}} & \text { if } k=0 ; \\
\text { (ii) } & 0 & \text { if } 0<k<2^{\tau} \\
\text { (iii) } & x^{2^{\tau}} \sum_{j=1}^{n} x_{j}^{2^{\tau}} & \text { if } k=2^{\tau} .
\end{array}\right.
$$

Proof. (i) is trivial. For (ii) and (iii) use induction on $n$, noting the fact that the one variable case is consistent with Lemma 1.4 in each case.

We prove even more general results. 
Lemma 3.2.

$$
S q^{k}\left(\boldsymbol{x}^{\boldsymbol{m}\left(2^{\tau}\right)}\right)=\left\{\begin{array}{lll}
\text { (i) } & \boldsymbol{x}^{\boldsymbol{m}\left(2^{\tau}\right)} & \text { if } k=0 \\
\text { (ii) } & 0 & \text { if } 0<k<2^{\tau} \\
\text { (iii) } & 0 & \text { if } k=2^{\tau} \text { and } \\
& & \text { all the } m_{i} \text { even } \\
\text { (iv) } \boldsymbol{x}^{\boldsymbol{m}\left(2^{\tau}\right)} \sum_{j=1}^{h} x_{j}^{2^{\tau}} & \text { if } k=2^{\tau}, \\
& & m_{1}, m_{2}, \ldots, m_{h} \text { odd }, \\
& & \text { and other } m_{i} \text { 's even } .
\end{array}\right.
$$

(Lemma 3.1 is analogous to the parts (1) and (3) of Lemma 3.1 in [17], however, Lemma 3.2 is stronger.)

Proof. (i) is trivial. To prove (ii) expand the notation (4) as

$$
\boldsymbol{x}^{\boldsymbol{m}\left(2^{\tau}\right)}=(\overbrace{x_{1}^{2^{\tau}} \cdots x_{1}^{2^{\tau}}}^{m_{1} \text { times }} \overbrace{x_{2}^{2^{\tau}} \cdots x_{2}^{2^{\tau}}}^{m_{2}} \cdots \overbrace{x_{n}^{2^{\tau}} \cdots x_{n}^{2^{\tau}}}^{m_{n} \text { times }}) .
$$

Now the result follows from Lemma 3.1(ii) taking $n=m_{1}+m_{2}+\cdots+m_{n}$.

We prove (iii) by induction on $n$, the number of variables. In the one variable case, put $m_{1}=2 n_{1}$. Then, using (ii) we get $S q^{k}\left(x_{1}^{m_{1}\left(2^{\tau}\right)}\right)=0$. Assume now the result for smaller variables than $n$. Then

$$
\begin{aligned}
S q^{k}\left(\boldsymbol{x}^{\boldsymbol{m}\left(2^{\tau}\right)}\right)= & S q^{0}\left(x_{1}^{m_{1}\left(2^{\tau}\right)} x_{2}^{m_{2}\left(2^{\tau}\right)} \cdots x_{n-1}^{m_{n-1}\left(2^{\tau}\right)}\right) S q^{2^{\tau}}\left(x_{n}^{m_{n}\left(2^{\tau}\right)}\right) \\
& +S q^{2^{\tau}}\left(x_{1}^{m_{1}\left(2^{\tau}\right)} x_{2}^{m_{2}\left(2^{\tau}\right)} \cdots x_{n-1}^{m_{n-1}\left(2^{\tau}\right)}\right) S q^{0}\left(x_{n}^{m_{n}\left(2^{\tau}\right)}\right) \quad \text { (by (ii)) } \\
= & 0,
\end{aligned}
$$

by assumption.

Finally, we prove (iv) by induction, this time, on $h$. Let $m_{1}$ be odd and the other $m_{i}$ 's even. Then, from (ii) and (iii) it follows that

$$
S q^{k}\left(\boldsymbol{x}^{\boldsymbol{m}\left(2^{\tau}\right)}\right)=\boldsymbol{x}^{\boldsymbol{m}\left(2^{\tau}\right)} x_{1}^{2^{\tau}} .
$$

Now assume the result is true for smaller values than $h$. Then

$$
\begin{aligned}
S q^{k}\left(\boldsymbol{x}^{\boldsymbol{m}\left(2^{\tau}\right)}\right)= & S q^{0}\left(x_{h}^{m_{h}\left(2^{\tau}\right)}\right) S q^{2^{\tau}}\left(\prod_{h \neq i=1}^{n} x_{i}^{m_{i}\left(2^{\tau}\right)}\right) \\
& +S q^{2^{\tau}}\left(x_{h}^{m_{h}\left(2^{\tau}\right)}\right) S q^{0}\left(\prod_{h \neq i=1}^{n} x_{i}^{m_{i}\left(2^{\tau}\right)}\right) \quad(\text { by (ii) }) \\
= & x_{h}^{m_{h}\left(2^{\tau}\right)}\left(\prod_{h \neq i=1}^{n} x_{i}^{m_{i}\left(2^{\tau}\right)}\right)\left(\sum_{j=1}^{h-1} x_{j}^{2^{\tau}}\right) \\
& +x_{h}^{m_{h}\left(2^{\tau}\right)+2^{\tau}} \prod_{h \neq i=1}^{n} x_{i}^{m_{i}\left(2^{\tau}\right)} \text { (by assumption) }
\end{aligned}
$$


A NOTE ON THE UNSTABILITY CONDITIONS OF THE STEENROD SQUARES 911

$$
\begin{aligned}
& =\prod_{i=1}^{n} x_{i}^{m_{i}\left(2^{\tau}\right)}\left(\sum_{j=1}^{h-1} x_{j}^{2^{\tau}}+x_{h}^{2^{\tau}}\right) \\
& =x^{\boldsymbol{m}\left(2^{\tau}\right)} \sum_{j=1}^{h} x_{j}^{2^{\tau}} .
\end{aligned}
$$

Corollary 3.3. In our earlier notations

$$
\boldsymbol{x}^{\boldsymbol{\alpha}}=x_{1}^{\alpha_{1}} x_{2}^{\alpha_{2}} \cdots x_{n}^{\alpha_{n}}, \quad \boldsymbol{x}^{\boldsymbol{m}\left(2^{\tau}\right)}=x_{1}^{m_{1}\left(2^{\tau}\right)} x_{2}^{m_{2}\left(2^{\tau}\right)} \cdots x_{n}^{m_{n}\left(2^{\tau}\right)},
$$

where $\alpha_{i}, m_{i} \geq 0$ and $\tau>0$, assume in addition that $1 \leq \alpha_{i}<2^{\tau}$ for $1 \leq i \leq n$. Let also $0 \leq k<2^{\tau}$. Then

$$
S q^{k}\left(\boldsymbol{x}^{\boldsymbol{m}\left(2^{\tau}\right)} \boldsymbol{x}^{\boldsymbol{\alpha}}\right)=\boldsymbol{x}^{\boldsymbol{m}\left(2^{\tau}\right)} S q^{k}\left(\boldsymbol{x}^{\boldsymbol{\alpha}}\right) .
$$

If, in addition, for $1 \leq h \leq n$, we assume $m_{1}, m_{2}, \ldots, m_{h}$ are odd and other $m_{i}$ 's even, then

$$
\begin{aligned}
S q^{2^{\tau}}\left(\boldsymbol{x}^{\boldsymbol{m}\left(2^{\tau}\right)} \boldsymbol{x}^{\boldsymbol{\alpha}}\right) & =\boldsymbol{x}^{\boldsymbol{m}\left(2^{\tau}\right)} S q^{2^{\tau}}\left(\boldsymbol{x}^{\boldsymbol{\alpha}}\right)+\boldsymbol{x}^{\boldsymbol{m}\left(2^{\tau}\right)} \boldsymbol{x}^{\boldsymbol{\alpha}} \sum_{j=1}^{h} x_{j}^{2^{\tau}} \\
S q^{k+2^{\tau}}\left(\boldsymbol{x}^{\boldsymbol{m}\left(2^{\tau}\right)} \boldsymbol{x}^{\boldsymbol{\alpha}}\right) & =\boldsymbol{x}^{\boldsymbol{m}\left(2^{\tau}\right)} \sum_{j=1}^{h} x_{j}^{2^{\tau}} S q^{k}\left(\boldsymbol{x}^{\boldsymbol{\alpha}}\right) .
\end{aligned}
$$

In particular, if all $m_{i}$ 's are even, then

$$
\begin{aligned}
S q^{2^{\tau}}\left(\boldsymbol{x}^{\boldsymbol{m}\left(2^{\tau}\right)} \boldsymbol{x}^{\boldsymbol{\alpha}}\right) & =\boldsymbol{x}^{\boldsymbol{m}\left(2^{\tau}\right)} S q^{2^{\tau}}\left(\boldsymbol{x}^{\boldsymbol{\alpha}}\right), \\
S q^{k+2^{\tau}}\left(\boldsymbol{x}^{\boldsymbol{m}\left(2^{\tau}\right)} \boldsymbol{x}^{\boldsymbol{\alpha}}\right) & =0 .
\end{aligned}
$$

Proof. (5) For $k=0$ the result is trivial. Let $1<k<2^{\tau}$. Then by Cartan formula

$$
S q^{k}\left(\boldsymbol{x}^{\boldsymbol{m}\left(2^{\tau}\right)} \boldsymbol{x}^{\boldsymbol{\alpha}}\right)=\boldsymbol{x}^{\boldsymbol{m}\left(2^{\tau}\right)} S q^{k}\left(\boldsymbol{x}^{\boldsymbol{\alpha}}\right)+\sum_{r=1}^{k} S q^{r}\left(\boldsymbol{x}^{\boldsymbol{m}\left(2^{\tau}\right)}\right) S q^{k-r}\left(\boldsymbol{x}^{\boldsymbol{\alpha}}\right) .
$$

But if $1 \leq r \leq k$, then $0<r<2^{\tau}$, and hence by Lemma 3.2(ii), we have $S q^{r}\left(\boldsymbol{x}^{\boldsymbol{m}\left(2^{\tau}\right)}\right)=0$. This proves (5).

To prove (6), by Lemma 3.2(ii) and Cartan formula we have

$$
S q^{2^{\tau}}\left(\boldsymbol{x}^{\boldsymbol{m}\left(2^{\tau}\right)} \boldsymbol{x}^{\boldsymbol{\alpha}}\right)=S q^{0}\left(\boldsymbol{x}^{\boldsymbol{m}\left(2^{\tau}\right)}\right) S q^{2^{\tau}}\left(\boldsymbol{x}^{\boldsymbol{\alpha}}\right)+S q^{2^{\tau}}\left(\boldsymbol{x}^{\boldsymbol{m}\left(2^{\tau}\right)}\right) S q^{0}\left(\boldsymbol{x}^{\boldsymbol{\alpha}}\right) .
$$

Now the result follows from Lemma 3.2(iv).

Finally, to prove (7) expand the left hand side of (7) using Cartan formula. Now, by Lemmas 3.2(ii) and 1.2(ii), we see that all terms in the expansion are zero except $S q^{2^{\tau}}\left(\boldsymbol{x}^{\boldsymbol{m}\left(2^{\tau}\right)}\right) S q^{k}\left(\boldsymbol{x}^{\boldsymbol{\alpha}}\right)$ which is the right hand side of (7) by Lemma 3.2.

The previous corollary shows clearly the main object stated at the beginning of this section. In the following example we illustrate all cases in Corollary 3.3; i.e., the relations (5) to (9). 


\section{Example 3.4.}

$$
\begin{aligned}
S q^{3}\left(x^{14} y^{11}\right) & =S q^{3}\left(x^{3 \cdot 2^{2}} y^{2 \cdot 2^{2}} x^{2} y^{3}\right)=x^{3 \cdot 2^{2}} y^{2 \cdot 2^{2}} S q^{3}\left(x^{2} y^{3}\right) \\
& =x^{12} y^{8}\left(x^{2} y^{6}+x^{4} y^{4}\right)=x^{14} y^{14}+x^{16} y^{12} \\
S q^{4}\left(x^{14} y^{11}\right) & =S q^{2^{2}}\left(x^{3 \cdot 2^{2}} y^{2 \cdot 2^{2}} x^{2} y^{3}\right)=x^{3 \cdot 2^{2}} y^{2 \cdot 2^{2}}\left(S q^{2^{2}}\left(x^{2} y^{3}\right)+x^{2} y^{3} \cdot x^{2^{2}}\right) \\
& =x^{12} y^{8}\left(x^{2} y^{7}+x^{4} y^{5}+x^{6} y^{3}\right)=x^{14} y^{15}+x^{16} y^{13}+x^{18} y^{11}, \\
S q^{7}\left(x^{14} y^{11}\right) & =S q^{3+2^{2}}\left(x^{3 \cdot 2^{2}} y^{2 \cdot 2^{2}} x^{2} y^{3}\right)=x^{3 \cdot 2^{2}} y^{2 \cdot 2^{2}} \cdot x^{2^{2}} S q^{3}\left(x^{2} y^{3}\right) \\
& =x^{16} y^{8}\left(x^{2} y^{6}+x^{4} y^{4}\right)=x^{18} y^{14}+x^{20} y^{12}, \\
S q^{4}\left(x^{18} y^{11}\right) & =S q^{2^{2}}\left(x^{4 \cdot 2^{2}} y^{2 \cdot 2^{2}} x^{2} y^{3}\right)=x^{4 \cdot 2^{2}} y^{2 \cdot 2^{2}} S q^{2^{2}}\left(x^{2} y^{3}\right) \\
& =x^{16} y^{8}\left(x^{2} y^{7}+x^{4} y^{5}\right)=x^{18} y^{15}+x^{20} y^{13}, \\
S q^{7}\left(x^{18} y^{11}\right) & =S q^{3+2^{2}}\left(x^{4 \cdot 2^{2}} y^{2 \cdot 2^{2}} x^{2} y^{3}\right)=0 .
\end{aligned}
$$

\section{The hit problem}

A homogeneous element $f$ of grading $d$ in a graded module $\mathbf{M}$ over $\mathcal{A}$ is called to be hit if it can be written as

$$
f=\sum_{k>0} S q^{k}\left(f_{k}\right)
$$

where the pre-image elements $f_{k}$ have degree less than $d$. The hit problem has first been studied by F. Peterson [14], R. Wood [23], W. Singer [18], S. Priddy [15] who show its relationship to several classical problems in cobordism theory, modular representation theory, Adams spectral theory for the stable homotopy of spheres, stable homotopy type of the classifying space of finite groups. The hit problem is to discover criteria for elements of $\mathbf{M}$ to be hit and find minimal generating sets for $\mathbf{M}$ as an $\mathcal{A}$-module. We cite to $[7,25]$ and the comprehensive reference [24] for detailed studies on the hit problem.

Since every $n$-variable polynomial over $\mathbb{F}_{2}$ is the sum of $n$-variable monomials, the following result is a direct consequence of Corollary 3.3. We adopt the notations of Corollary 3.3 and assume $f, g$ are $n$-variable polynomials.

Proposition 4.1. Let $\tau>0$ and $0 \leq k<2^{\tau}$.

(i) If $f$ is hit, then so is $g=\boldsymbol{x}^{\boldsymbol{m}\left(2^{\tau}\right)} f$.

(ii) Assume, in addition, that $m_{1}, m_{2}, \ldots, m_{h}$ are odd and other $m_{i}$ 's even for $1 \leq h \leq n$. If $f$ is hit, then so is $\boldsymbol{x}^{\boldsymbol{m}\left(2^{\tau}\right)} \sum_{j=1}^{h} x_{j}^{2^{\tau}} S q^{k}(f)$.

(iii) Assume, in particular, that all $m_{i}$ 's are even. If $f$ is hit, then so is $\boldsymbol{x}^{\boldsymbol{m}\left(2^{\tau}\right)} S q^{2^{\tau}}(f)$.

(Parts (i) and (iii) of Proposition 4.1 are consistent, respectively, to Theorems 3.3 and 3.4 of [17] in the notations there.) 
The converse of the statement (i) is true only if $g=x^{\boldsymbol{m}\left(2^{\tau}\right)} f$ is hit via

$$
g=\sum_{0<k<2^{\tau}} S q^{k}\left(\boldsymbol{x}^{\boldsymbol{m}\left(2^{\tau}\right)} f_{k}\right)
$$

Similar argument hold for the converse of the other statements.

Let the monomial $x^{\boldsymbol{\alpha}^{\prime}}$ be a permutation of the monomial $x^{\alpha}$. Then by the equation (5) in Corollary 3.3 we have

$$
S q^{k}\left[\boldsymbol{x}^{\boldsymbol{m}\left(2^{\tau}\right)}\left(\boldsymbol{x}^{\boldsymbol{\alpha}}+\boldsymbol{x}^{\boldsymbol{\alpha}^{\prime}}\right)\right]=\boldsymbol{x}^{\boldsymbol{m}\left(2^{\tau}\right)} S q^{k}\left(\boldsymbol{x}^{\boldsymbol{\alpha}}+\boldsymbol{x}^{\boldsymbol{\alpha}^{\prime}}\right) .
$$

Using this fact we can state the same results as Proposition 4.1 for symmetric polynomials. In particular, in Proposition 4.1 both $f$ and $g$ may be chosen symmetric if we take $\boldsymbol{x}^{\boldsymbol{m}\left(2^{\tau}\right)}$ symmetric $n$-variable, i.e.,

$$
\boldsymbol{x}^{\boldsymbol{m}\left(2^{\tau}\right)}=x_{1}^{m_{1}\left(2^{\tau}\right)} x_{2}^{m_{2}\left(2^{\tau}\right)} \cdots x_{n}^{m_{n}\left(2^{\tau}\right)},
$$

where $m_{1}\left(2^{\tau}\right)=\cdots=m_{n}\left(2^{\tau}\right)>0$. If this is the case, $f_{k}$ will be symmetric, as well. For the symmetric hit problem we cite to $[9,10]$.

\section{Further consequences}

In this section we get some further tools in handling with the Steenrod squares in the 2 -variable case and, since higher variables are determined inductively, these tools apply for general $n$.

Proposition 5.1. Given $\tau \geq 1$, let

(i) $0 \leq \alpha<2^{\tau}$;

(ii) $2^{\tau} \leq \beta<2^{\tau+1}-1$;

(iii) $2^{\tau}<\alpha+\beta<2^{\tau+1}$.

Then

$$
S q^{2^{\tau}}\left(x^{\alpha} y^{\beta}\right)=x^{\alpha} y^{\beta+2^{\tau}}
$$

Proof. Put $\beta=\beta^{\prime}+2^{\tau}$, where $0 \leq \beta^{\prime} \leq 2^{\tau}-1$. By Cartan formula and Lemma 1.3 we have

$$
\begin{aligned}
S q^{2^{\tau}}\left(x^{\alpha} y^{\beta}\right) & =S q^{0}\left(x^{\alpha}\right) S q^{2^{\tau}}\left(y^{\beta}\right)+\sum_{r=1}^{2^{\tau}} S q^{r}\left(x^{\alpha}\right) S q^{2^{\tau}-r}\left(y^{\beta}\right) \\
& =x^{\alpha} y^{\beta+2^{\tau}}+y^{2^{\tau}} \sum_{r=1}^{2^{\tau}} S q^{r}\left(x^{\alpha}\right) S q^{2^{\tau}-r}\left(y^{\beta^{\prime}}\right) .
\end{aligned}
$$

On the other hand,

$$
\begin{aligned}
\sum_{r=1}^{2^{\tau}} S q^{r}\left(x^{\alpha}\right) S q^{2^{\tau}-r}\left(y^{\beta^{\prime}}\right)= & \sum_{1 \leq r \leq \alpha} S q^{r}\left(x^{\alpha}\right) S q^{2^{\tau}-r}\left(y^{\beta^{\prime}}\right) \\
& +\sum_{\alpha<r \leq 2^{\tau}} S q^{r}\left(x^{\alpha}\right) S q^{2^{\tau}-r}\left(y^{\beta^{\prime}}\right) .
\end{aligned}
$$


If $r \leq \alpha, 2^{\tau}-r>\beta^{\prime}$ and hence $S q^{2^{\tau}-r}\left(y^{\beta^{\prime}}\right)=0$, and if $r>\alpha, S q^{r}\left(x^{\alpha}\right)=0$. Therefore,

$$
\sum_{r=1}^{2^{\tau}} S q^{r}\left(x^{\alpha}\right) S q^{2^{\tau}-r}\left(y^{\beta^{\prime}}\right)=0,
$$

and the proof is completed.

Proposition 5.2. Let $m \geq n+2, n \geq 1$. Let

(i) $\quad 2^{m-2} \leq \alpha \leq 2^{m-2}+2^{n-1}-1$;

(ii) $2^{m-2}+2^{n-1}-1 \leq \beta \leq 2^{m-2}+2^{n}-2$;

(iii) $\alpha+\beta=2^{m-1}+2^{n}-2$.

Then

$$
S q^{2^{m-1}}\left(x^{\alpha} y^{\beta}\right)=\left(x^{\alpha+2^{m-2}} y^{\beta+2^{m-2}}\right) .
$$

Proof. By Cartan formula we have

$$
S q^{2^{m-1}}\left(x^{\alpha} y^{\beta}\right)=\sum_{0 \leq r<2^{m-1}-\beta} S q^{r}\left(x^{\alpha}\right) S q^{2^{m-1}-r}\left(y^{\beta}\right) .
$$

If $0 \leq r<2^{m-1}-\beta$, then $2^{m-1}-r>\beta$ and $S q^{2^{m-1}-r}\left(y^{\beta}\right)=0$.

If $2^{m-1}-\beta \leq r<2^{m-2}$, then $r>\alpha-2^{m-2}$. Hence, by Corollary 3.3,

$$
S q^{r}\left(x^{\alpha}\right)=S q^{r}\left(x^{2^{m-2}} x^{\alpha-2^{m-2}}\right)=0 .
$$

If $2^{m-2}<r \leq \alpha$, then $2^{m-1}-r>\beta-2^{m-2}$. Once again,

$$
S q^{2^{m-1}-r}\left(y^{\beta}\right)=S q^{2^{m-1}-r}\left(y^{2^{m-2}} y^{\beta-2^{m-2}}\right)=0 .
$$

Finally, if $\alpha<r \leq 2^{m-1}$, then $S q^{r}\left(x^{\alpha}\right)=0$. So, by splitting the summation, one sees that only the middle term is non-zero. Thus,

$$
S q^{2^{m-1}}\left(x^{\alpha} y^{\beta}\right)=S q^{2^{m-2}}\left(x^{\alpha}\right) S q^{2^{m-2}}\left(y^{\beta}\right)=\left(x^{\alpha+2^{m-2}} y^{\beta+2^{m-2}}\right) .
$$

\section{The $S q$-matrices}

The subject of this section is to introduce some particular matrices, which we call them $S q$-matrices, and apply them to simplify the action of the Steenrod squares, as explained in Section 2. To do this, we need some preliminaries.

Definition 6.1. Let $M$ be an $m \times n$ matrix. By a reverse transpose of $M$, denoted $M^{r t}$, we mean an $n \times m$ matrix obtained by reversing the order of rows of $M^{t}$, the transpose of $M$. Therefore,

$$
M_{j i}^{r t}=M_{(n+1-j) i}^{t}=M_{i(n+1-j)}
$$

for $1 \leq i \leq m, 1 \leq j \leq n$. The following result follows directly from the definition.

Proposition 6.2. Given any $m \times n$ matrix $M$, the product $M M^{r t}$ is a symmetric $m \times m$ matrix. 
The next lemma in [6] describe how binomial coefficients can be computed modulo a prime.

Lemma 6.3. If $p$ is a prime, then $\left(\begin{array}{c}m \\ n\end{array}\right)=\prod_{i}\left(\begin{array}{c}m_{i} \\ n_{i}\end{array}\right) \bmod p$, where $m=\sum_{i} m_{i} p^{i}$ and $n=\sum_{i} n_{i} p^{i}$, with $0 \leq m_{i}<p$ and $0 \leq n_{i}<p$, are the $p$-adic expansions of $m$ and $n$.

When $n=2$, for example, the extreme cases of a dyadic expansion consisting of a single 1 or all 1's give

$$
\begin{aligned}
S q\left(x^{2^{k}}\right) & =x^{2^{k}}+x^{2^{k+1}} \\
S q\left(x^{2^{k}-1}\right) & =x^{2^{k}-1}+x^{2^{k}}+x^{2^{k}+1}+\cdots+x^{2^{k+1}-2}
\end{aligned}
$$

for all $x$ with degree 1 . More generally, the coefficient of $S q\left(x^{n}\right)$ can be read off from the $(n+1)$-th row of the mod 2 Pascal triangle, a portion of which is shown in the Figure 1, where dots denote zeros [6].

Definition 6.4. Let $m$ be a positive integer. For $1 \leq k \leq 2^{m}-1$ the Sq-matrix $\mathcal{S}_{k}$ is a $2^{m} \times(k+1)$ matrix defined by

$$
\left(\mathcal{S}_{k}\right)_{i j}=S q^{j-1}\left(x^{i}\right) .
$$

In other words, the terms of $S q\left(x^{n}\right)$ can be read off from the $n$-th row of $\mathcal{S}_{k}$.

Without any confusion, if convenient, in expression of $S q$-matrices each non zero entry may be denoted just by the power of $x$ in it. Figure 2 shows the $32 \times 32 S q$-matrix $\mathcal{S}_{31}$, where, as Figure 1, dots denote zeros. Note that it contains the square $S q$-matrices $\mathcal{S}_{15}, \mathcal{S}_{7}, \mathcal{S}_{3}$, and $\mathcal{S}_{1}$ as bisecting sub-blocks.

As seen, comparing two Figures 1 and 2, if we remove the top row of Figure 1 then, up to arrangement, the position of zeros in both figures are the same.

In the following algorithm, given a positive integer $m$, we construct the $2^{m} \times 2^{m} S q$-matrix $\mathcal{S}_{2^{m}-1}$ using Corollary 3.3. For $1 \leq k \leq 2^{m}-1$ the $S q$ matrix $\mathcal{S}_{k}$ can be obtained from $\mathcal{S}_{2^{m}-1}$ by choosing the first $k+1$ columns.

Algorithm 6.5. 1) Define

$$
\mathcal{S}_{1}=\left[\begin{array}{ll}
1 & 2 \\
2 & 0
\end{array}\right]
$$

2) For $t=1$ to $m-1$ do

2.1) For $i, j=1$ to $2^{t}$ do

2.1.1) Define $\mathcal{T}_{i j}=\left(\mathcal{S}_{2^{t}-1}\right)_{i j}+2^{t}$

2.1.2) Define $\mathcal{U}_{i j}=\left(\mathcal{S}_{2^{t}-1}\right)_{i j}+2^{t+1}$;

2.1.3) Define 0 to be the $2^{t} \times 2^{t}$ zero matrix;

2.2) Define the $2^{t+1} \times 2^{t+1}$ matrix

$$
\mathcal{S}=\left[\begin{array}{c|c}
\mathcal{S}_{2^{t}-1} & \mathbf{0} \\
\hline \mathcal{T} & \mathcal{U}
\end{array}\right] ;
$$




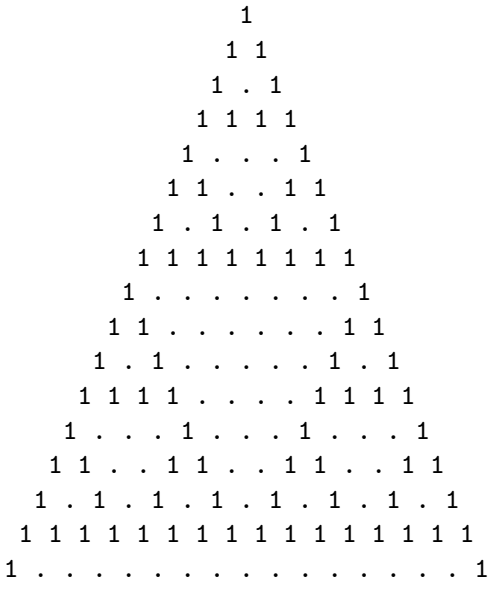

Figure 1: Mod 2 Pascal triangle

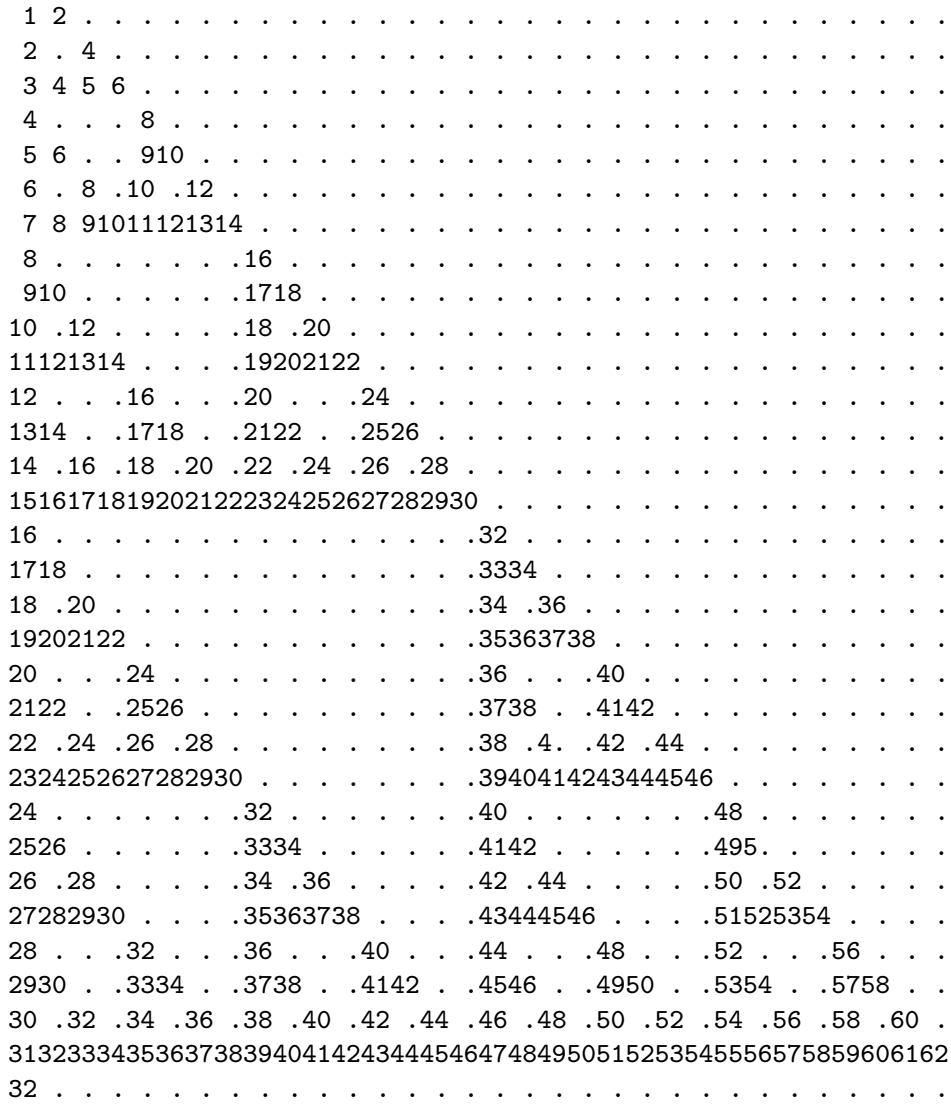

Figure 2: The $S q$-matrix $\mathcal{S}_{31}$ 
2.3) Define $\mathcal{S}_{2^{t+1}-1}$ to be the matrix obtained from $\mathcal{S}$ by substituting $\mathbf{0}_{2^{t}}$ by $2^{t}$, and $\mathcal{U}_{2^{t} 1}$ by 0 .

The following observation follows directly from Definition 6.4 and Cartan formula where, in $\mathcal{S}_{k}^{r t}$ the symbol $y$ is used instead of $x$.

Proposition 6.6. Let $m$ be a positive integer and $1 \leq k \leq 2^{m}-1$. Then

$$
\left(\mathcal{S}_{k} \mathcal{S}_{k}^{r t}\right)_{i j}=S q^{k}\left(x^{i} y^{j}\right), \quad 1 \leq i, j \leq 2^{m} .
$$

\section{Problems}

There are lots of polynomial algebras over the Steenrod algebra which admits unstable operations satisfy the Cartan formula: the well-known one is the Dickson algebra [3, 20], and the interesting one is the recent work of Singer [19] which is a bigraded Steenrod algebra $\mathcal{H}$ over a graded vector space. The generators $\left\{\mathbb{S} q^{l}, l \geq 0\right\}$ of this algebra $\mathcal{H}$, like the generators of the classic Steenrod algebra, satisfy the Cartan formula and have unstable properties. The challenge problem on these type of algebras is to find some easier tools to compute them, say, by matrix algebras like Proposition 6.6.

On the other hand, there is a correspondence [12] between unstable $\mathcal{A}$ algebras and unstable $\mathcal{K}$-algebras, where $\mathcal{K}$ is the mod 2 Kudo-Araki-May algebra which is the $\mathbb{F}_{2}$-bialgebra generated by elements $\left\{D_{i}, i \geq 0\right\}$, bigraded by length and topological degree $\left(\left|D_{i}\right|=i\right)$. This correspondence completely determined by iterating the conversion formulae: On any even $x_{l}$ of degree $l$, and for all $j \geq 0$, one has

$$
D_{j} x_{l}=S q^{l-j} \text { equivalently } S q^{j} x_{l}=D_{l-j} x_{l} .
$$

A natural question is to find some computational tools for the corresponding operations.

More generally, wherever an unstable operation satisfies the Cartan formula, the same question arises.

\section{References}

[1] J Adem, The iteration of the Steenrod squares in algebraic topology, Proc. Nat. Acad. Sci. U. S. A. 38 (1952), 720-726.

[2] H. Cartan, Une theórie axiomatique des carrés de Steenrod, C. R. Acad. Sci. Paris 230 (1950), 425-427.

[3] L. E. Dickson, A fundamental system of invariants of the general modular linear group with a solution of the form problem, Trans. Amer. Math. Soc. 12 (1911), no. 1, 75-98.

[4] J. Dieudonné, A History of Algebraic and Differential Topology, 1900-1960, Birkhauser Boston, Inc., Boston, MA, 1989.

[5] J. R. Harper, Secondary Cohomology Operations, Graduate Studies in Mathematics, 49. American Mathematical Society, Providence, RI, 2002.

[6] A. Hatcher, Algebraic Topology, Cambridge University Press, 2002.

[7] A. S. Janfada, The hit problem for symmetric polynomials over the Steenrod algebra, Thesis, Manchester University, 2000.

[8] _ A criterion for a monomial in $\mathbf{P}(3)$ to be hit, Math. Proc. Camb. Phil. Soc. 145 (2008), 587-599. 
[9] A. S. Janfada and R. M. W. Wood, The hit problem for symmetric polynomials over the Steenrod algebra, Math. Proc. Cambridge Philos. Soc. 133 (2002), 295-303.

[10] Generating $H^{*}\left(\mathrm{BO}(3), \mathbb{F}_{2}\right)$ as a module over the Steenrod algebra, Math. Proc. Cambridge Philos. Soc. 134 (2003), 239-258.

[11] R. E. Mosher and M. C. Tangora, Cohomology operations and applications in homotopy theory, Harper \& Row, Publishers, New York-London, 1968.

[12] D. J. Pengelley and F. Williams, Global structure of the mod two symmetric algebra, $H^{*}\left(B \mathrm{O} ; \mathbb{F}_{2}\right)$, over the Steenrod algebra, Algebr. Geom. Topol. 3 (2003), 1119-1138.

[13] Sheared algebra maps and operation bialgebras for mod 2 homology and cohomology, Trans. Amer. Math. Soc. 352 (2000), no. 4, 1453-1492.

[14] F. P. Peterson, Generators of $H^{*}(\mathbb{R} \mathbf{P} \vee \mathbb{R} \mathbf{P})$ as a module over the Steenrod algebra, Abstracts Amer. Math. Soc. 123 (1995), 627-637.

[15] S. Priddy, On characterizing summands in the classifying space of a group. I, Amer. J. Math. 112 (1990), no. 5, 737-748.

[16] J.-P. Serre, Cohomologie modulo 2 des complexes d'Eilenberg-MacLane, Comment. Math. Helv. 27 (1953), 198-232.

[17] J. Silverman and W. M. Singer, On the action of Steenrod squares on polynomial algebras. II, J. Pure Appl. Algebra 98 (1995), no. 1, 95-103.

[18] W. M. Singer, The transfer in homological algebra, Math. Z. 202 (1989), no. 4, 493-523.

[19] _ Rings of symmetric functions as modules over the Steenrod algebra, Algebraic \& Geometric Topology 8 (2008), 541-562.

[20] L. Smith and R. M. Switzer, Realizability and nonrealizability of Dickson algebras as cohomology rings, Proc. Amer. Math. Soc. 89 (1983), no. 2, 303-313.

[21] N. E. Steenrod, Products of cocycles and extensions of mappings, Ann. of Math. (2) 48 (1947), 290-320.

[22] N. E. Steenrod and D. B. A. Epstein, Cohomology operations, Princeton University Press, 1962.

[23] R. M. W. Wood, Modular representations of $\operatorname{GL}\left(n, F_{p}\right)$ and homotopy theory, Algebraic topology, Göttingen 1984, 188-203, Lecture Notes in Math., 1172, Springer, Berlin, 1985.

[24] _ Problems in the Steenrod algebra, Bull. London Math. Soc. 30 (1998), no. 5, $449-517$.

[25] _ Steenrod squares of polynomials and the Peterson conjecture, Math. Proc. Cambridge Philos. Soc. 105 (1989), no. 2, 307-309.

Department of Mathematics

URMIA UNIVERSITY

P. O. Box 165, URMiA, IrAN

E-mail address: a.sjanfada@mail.urmia.ac.ir 